\title{
Autopercepção e conhecimento em saúde bucal de Agentes Comunitários de Saúde de um município baiano, Brasil
}

Self-perception and knowledge in oral health of Community Health Agents of a municipality in Bahia, Brazil Autopercepción y conocimiento en salud bucal de Agentes Comunitarios de Salud de un municipio bahian, Brasil

Inglide da Silveira PEREIRA ${ }^{1}$

Fábio Silva de CARVALHO²

Cristiane Alves Paz de CARVALHO

${ }^{1}$ Cirurgiã-Dentista, pela Universidade Estadual do Sudoeste da Bahia, UESB, Departamento de Saúde I, Curso de Odontologia, 45208-409 Jequié-BA, Brasil

${ }^{2}$ Doutor em Ciências, Professor Adjunto da Universidade Estadual do Sudoeste da Bahia, UESB, Departamento de Saúde I, Curso de Odontologia, 45208-409 Jequié-BA, Brasil

${ }^{3}$ Doutora em Ciências, Professora Adjunta da Universidade Estadual do Sudoeste da Bahia, UESB, Departamento de Saúde I, Curso de Odontologia, 45208-409 Jequié-BA, Brasil

\section{Resumo}

Objetivos: Avaliar a autopercepção e o conhecimento em saúde bucal do Agente Comunitário de Saúde (ACS). Material e método: Estudo realizado no município de Cruz das Almas-BA com 127 ACS, de 15 unidades de saúde, das quais 12 apresentam equipes de saúde bucal. Foram utilizados dois questionários de abordagem quantitativa. Resultados: Aproximadamente $87,0 \%$ dos ACS afirmaram necessitar de tratamento de seus dentes ou gengivas e classificaram como regulares tanto sua saúde bucal (64,6\%) quanto a aparência dos seus dentes e gengivas (60,6\%). Cerca de 90,0\% respondeu que a educação em saúde bucal é atribuição do ACS, embora 55,9\% não realizem ou fazem orientação apenas eventualmente, durante as visitas. Conclusões: Os ACS mostraram-se críticos quanto a própria saúde bucal e apresentaram conhecimento adequado. Verificou-se a necessidade de realizar educação continuada em saúde bucal, para que os ACS se tornem mais capacitados e seguros nas orientações e encaminhamento dos usuários ao serviço de saúde.

Descritores: Estratégia Saúde da Família; Agentes Comunitários de Saúde; Saúde Bucal.

Abstract

Objectives: To evaluate the self-perception and the Community Health Agent (CHA) knowledge about oral health. Material and method: A study was conducted in Cruz das Almas BA with 127 ACS, from 15 health units, of which 12 have oral health teams. Two quantitative approach questionnaires were used. Results: Approximately $87.0 \%$ of CHA said need treatment of their teeth or gums and classified as regular both your oral health (64.6\%) and the appearance of your teeth and gums (60.6\%). About $90.0 \%$ said that oral health education is the responsibility of $\mathrm{CHA}$, while $55.9 \%$ do not perform or make orientation only occasionally during visits. Most replied that the baby's mouth hygiene should be performed even when it still has no teeth $(95.3 \%)$ and that children should use toothpaste with fluoride (58.3\%). For most of CHA, pregnant women (92.1\%) and diabetic $(94.8 \%)$ can pass through the dental care. Conclusions: The $\mathrm{CHA}$ have shown critical view as their oral health and had adequate knowledge. However, there is the need for continuing education in oral health, so that $\mathrm{CHA}$ become more empowered and safe in the guidelines and referral of users to the health service.

Descriptors: Family Health Strategy; Community Health Workers; Oral Health.

\section{Resumen}

Objetivos: Evaluar la autopercepción y el conocimiento en salud bucal del Agente Comunitario de Salud (ACS). Material y método: Estudio realizado en el municipio de Cruz das Almas-BA con 127 ACS, de 15 unidades de salud, de las cuales 12 presentan equipos de salud bucal. Se utilizaron dos cuestionarios de enfoque cuantitativo. Resultados: Aproximadamente el $87,0 \%$ de los ACS afirmaron necesitar tratamiento de sus dientes o encías y clasificaron como regulares tanto su salud bucal $(64,6 \%)$ como la apariencia de sus dientes y encías $(60,6 \%)$. Cerca del 90,0\% respondió que la educación en salud bucal es atribución del ACS, aunque el 55,9\% no realiza o hace orientación sólo eventualmente, durante las visitas. Conclusiones: Los ACS se mostraron críticos en cuanto a la propia salud bucal y presentaron un conocimiento adecuado. Se verificó la necesidad de realizar educación continuada en salud bucal, para que los ACS se vuelvan más capacitados y seguros en las orientaciones y encaminamiento de los usuarios al servicio de salud.

Descriptores: Estrategia de Salud Familiar; Agentes Comunitarios de Salud; Salud Bucal.

INTRODUÇÃO

A Estratégia Saúde da Família (ESF) caracteriza-se como importante meio para a reorganização da Atenção Primária à Saúde (APS), de acordo com os preceitos do Sistema Único de Saúde (SUS) ${ }^{1}$. O termo "Atenção Primária à Saúde" compreende um conjunto bastante diversificado de atividades clínicas de baixa densidade tecnológica, em espaços onde se dá, majoritariamente, o primeiro contato dos indivíduos com o sistema de saúde. Estes espaços devem ser capacitados para a resolução da grande maioria dos problemas de saúde da população, bem como, fazer a referência para os outros níveis de atenção à saúde ${ }^{2}$.

Como parte do processo de reforma do setor saúde, em 1991, foi criado o Programa de Agentes Comunitários de Saúde (PACS), com o intuito de contemplar a prevenção, a promoção da saúde e a ampliação do acesso ao sistema de saúde ${ }^{3}$. Em 1994, como forma de reorientação do modelo assistencial, houve a criação do Programa de Saúde da Família (PSF) pelo Ministério da Saúde (MS), com integralidade nas ações para proteção e promoção da saúde da família ${ }^{4}$. O Ministério da Saúde, reconhecendo a importância do PACS e do PSF, em 1997, redirecionou a assistência ambulatorial e domiciliar para consolidação do SUS, regulamentou as diretrizes desses programas e aprovou suas formas de implantação e operacionalização, por meio da Portaria federal $n^{0}$ 1886/19975.

Com a criação do PSF, reafirmaram-se os princípios doutrinários e organizativos do SUS, com o objetivo de substituir o modelo 
vigente de assistência à saúde, que visava ao atendimento da demanda espontânea, eminentemente curativo, hospitalocêntrico, de alto custo, sem redes hierarquizadas por complexidade e sem estabelecimento de vínculos $^{3,6}$. Desta forma, o atendimento na Unidade Básica de Saúde (UBS) ou no domicílio passou a ser efetuado pelas equipes de Saúde da Família, com a família sendo percebida em seu ambiente físico e social, observando o indivíduo como um todo, vivenciando desta forma uma rotina que vai além das práticas curativas.

As equipes mínimas de Saúde da Família são compostas por médico generalista ou médico familiar, enfermeiro, auxiliar ou técnico de enfermagem e agentes comunitários de saúde $(A C S)^{1}$. Tendo em vista a necessidade em aumentar 0 acesso da população às ações de saúde bucal, a Portaria GM/MS no 1.444, de 28 de dezembro de 2000 regulamentou e estabeleceu incentivo financeiro destinado a melhorias da atenção básica à saúde bucal prestada nos municípios. A partir de então, as Equipes de Saúde Bucal (ESB) passaram a ser parte integrante da Estratégia Saúde da Família (ESF) 7,8 .

As ESB objetivam a ampliação do acesso da população brasileira às ações de promoção e recuperação da saúde bucal, bem como a prevenção de doenças e agravos relacionados; melhoria dos índices epidemiológicos de saúde bucal e reorganização das ações de saúde bucal no âmbito da Atenção Básica ${ }^{9}$.

As diretrizes para o exercício profissional dos ACS foram explicitadas pelo decreto n. 3189/1999 ${ }^{10}$. Entretanto, somente em 2002 criou-se a profissão de ACS, a partir da lei $n .{ }^{\circ}$ $10507 / 2002$, que estabelece o seu exercício apenas no âmbito do SUS ${ }^{11}$. Os ACS, como parte fundamental das equipes de Saúde da Família, realizam a adscrição de famílias em base geográfica definida e são responsáveis pelo acompanhamento de no máximo 750 pessoas ${ }^{1}$. Têm como um dos objetivos principais no desenvolvimento do seu trabalho, o de acompanhar gestantes, crianças e pacientes com alguma patologia crônica ${ }^{12}$. Por estarem mais próximos da comunidade atendida, as possibilidades de efetividade das ações e estratégias são maiores, no que se refere à prevenção de doenças e promoção de saúde ${ }^{13}$.

A atuação do ACS aumenta a capacidade da população em entender determinadas informações e a construir um conhecimento em saúde e, consequentemente, aumenta suas habilidades no controle de determinantes em saúde. Assim, o "saber" do ACS em saúde bucal, de forma adequada, torna-se essencial; pois os agentes identificam as famílias mais vulneráveis e que necessitam de atenção mais específica. Desta forma, colaboram com a equipe de saúde bucal e possibilitam melhorias no acesso dos usuários, além de evitar atendimentos tardios e ajudar a reduzir, portanto, os atendimentos de urgência ${ }^{14}$.

O presente estudo teve como objetivo verificar a autopercepção e o conhecimento em saúde bucal dos Agentes Comunitários de Saúde de um município baiano.

MATERIAL E MÉTODO

Foi realizado um estudo transversal, de natureza quantitativa no município de Cruz das Almas, situado no Recôncavo Sul da Bahia, a cerca de 146 quilômetros da capital do estado, com uma população em torno de 63.761 habitantes e densidade demográfica de 402,12 hab. $/ \mathrm{km}^{15}$. A cidade conta com 15 Unidades de Saúde, assim distribuídas: 13 Unidades de Saúde da Família (USF), sendo sete localizadas na zona urbana (seis delas apresentam ESB) e seis na zona rural (todas com ESB); além de duas Unidades Básicas de Saúde (UBS), localizadas na zona urbana e que não apresentam ESB.

A população deste estudo foi composta pelos 149 ACS atuantes em todas as Unidades de Saúde, considerando-se, portanto, as USF e as UBS. A amostra final foi composta por 127 ACS, pois nos períodos de coleta, alguns agentes encontravam-se de férias, licença maternidade, não compareceram nos dias de coleta ou recusaram-se a participar da pesquisa.

Foram utilizados dois questionários de abordagem quantitativa. O primeiro, composto por sete questões, referentes à autopercepção em saúde bucal ${ }^{16}$. O segundo, elaborado pelos pesquisadores, refere-se à caracterização sociodemográfica dos ACS, bem como a investigação dos seus conhecimentos sobre saúde bucal.

A coleta de dados desta pesquisa teve início após a autorização concedida pela Secretaria Municipal de Saúde do Município e a aprovação pelo Comitê de Ética em Pesquisa da Universidade Estadual do Sudoeste da Bahia (CEP/UESB), (CAAE 43795415.8.0000.0055), bem como após a autorização do ACS por meio da assinatura do Termo de Consentimento Livre e Esclarecido.

Os dados foram tabulados em planilha 
do programa Office Excel $2016 \AA$. Foi realizada estatística descritiva, baseada em proporções e frequências absoluta e relativa simples para caracterização da amostra e análise dos resultados.

\section{RESULTADOS}

Dos 149 ACS atuantes no município, participaram da pesquisa 127, o que corresponde a uma taxa de participação de $85,2 \%$. A maioria dos ACS desse estudo é do sexo feminino $(81,1 \%)$, acompanha mais de 100 famílias $(78,0 \%)$, apresenta $2^{\circ}$ grau completo $(74,8 \%)$, tem renda familiar entre um e três salários mínimos $(50,4 \%)$ e exerce a profissão há mais de 10 anos (62,2\%) (Tabela 1). Na Tabela 2, são apresentados os dados referentes à autopercepção do ACS quanto à saúde bucal. $\mathrm{Na}$ Tabela 3 estão os dados relacionados à capacitação, atividades em saúde bucal e autoavaliação do conhecimento. A Tabela 4 traz informações a respeito do conhecimento e conduta dos ACS em relação à saúde bucal.

Tabela 1. Caracterização demográfica e socioeconômica dos ACS, Cruz da Almas-BA, Brasil - 2015

\begin{tabular}{|c|c|c|}
\hline Variável & $\mathbf{N}$ & $\%$ \\
\hline \multicolumn{3}{|l|}{ Local da unidade } \\
\hline & 91 & 71,7 \\
\hline Rural & 36 & 28,3 \\
\hline \multicolumn{3}{|l|}{ Sexo } \\
\hline Feminino & 103 & 81,1 \\
\hline \multirow{2}{*}{\multicolumn{3}{|c|}{ Número de famílias que acompanha }} \\
\hline & & \\
\hline$<100$ & 18 & 14,2 \\
\hline $\begin{array}{l}>100 \\
\text { Não responderam }\end{array}$ & 99 & 78,0 \\
\hline \multicolumn{3}{|l|}{ Escolaridade } \\
\hline $2^{\circ}$ grau completo & 95 & 74,8 \\
\hline $2^{\circ}$ grau incompleto & 8 & 6,3 \\
\hline Superior completo & 10 & \\
\hline Superior incompleto & 14 & 11,0 \\
\hline \multicolumn{3}{|l|}{ Renda Familiar } \\
\hline Menos de 1 salário mínimo & 1 & 0,8 \\
\hline 1 salário mínimo & 48 & \\
\hline Entre 1 e 3 salários mínimos & 64 & 50,4 \\
\hline Mais de 3 salários mínimos & 9 & 7,1 \\
\hline \multirow{2}{*}{\multicolumn{3}{|c|}{ Tempo de trabalho como ACS }} \\
\hline & & \\
\hline $1-2$ anos & 1 & 0,8 \\
\hline 3-4 anos & 20 & 15,7 \\
\hline $5-7$ anos & 8 & 6,3 \\
\hline $8-10$ anos & 19 & 15,0 \\
\hline Mais de 10 anos & $\begin{array}{r}79 \\
127\end{array}$ & $\frac{62,2}{100,0}$ \\
\hline
\end{tabular}

Tabela 2. Autopercepção em saúde bucal dos ACS, Cruz da Almas-BA, Brasil - 2015

\begin{tabular}{|c|c|c|}
\hline \multirow{2}{*}{\multicolumn{3}{|c|}{\begin{tabular}{l|l} 
Variável & $\mid$ \\
\end{tabular}}} \\
\hline & & \\
\hline Sim & 110 & 86,6 \\
\hline & 17 & 13,4 \\
\hline \multicolumn{3}{|l|}{ Classificação da Saúde Bucal } \\
\hline Regular & 82 & 64,6 \\
\hline & 10 & 7,9 \\
\hline \multicolumn{3}{|l|}{ Aparência dos dentes/gengiva } \\
\hline $\begin{array}{ll}\text { Boa } \\
\text { Reoplar }\end{array}$ & 39 & 30,7 \\
\hline Regular & 78 & $\frac{61,4}{70}$ \\
\hline \multirow{2}{*}{\multicolumn{3}{|c|}{ Classificação da mastigação }} \\
\hline Boa & & \\
\hline Regular & 68 & 32,3 \\
\hline & 18 & 14,2 \\
\hline \multicolumn{3}{|l|}{ Classificação da fala } \\
\hline Boa & 76 & 59,8 \\
\hline Regular & 44 & 34,6 \\
\hline Ruim & 7 & $5,5,5>0$ \\
\hline \multirow{2}{*}{\multicolumn{3}{|c|}{ Aparência de dentes/gengivas e relacionamento }} \\
\hline & & 25,2 \\
\hline Sim, afeta moderadamente & 10 & \\
\hline Sim, afeta muito & 4 & \\
\hline $\begin{array}{l}\text { Não afeta } \\
\text { Dor em dentes/gengiva nos últimos } 6 \mathrm{~m}\end{array}$ & leses & 63,8 \\
\hline \multicolumn{3}{|c|}{ Dor em dentes/gengiva nos últimos 6 meses } \\
\hline Pouca dor & 14 & 11,0 \\
\hline Dor moderada & 12 & 9,4 \\
\hline Muita dor & 2 & 1,6 \\
\hline Total & 127 & 100,0 \\
\hline
\end{tabular}

Tabela 3. Capacitação, atribuição para educação em saúde bucal e auto avaliação do conhecimento, Cruz da Almas-BA, Brasil - 2015

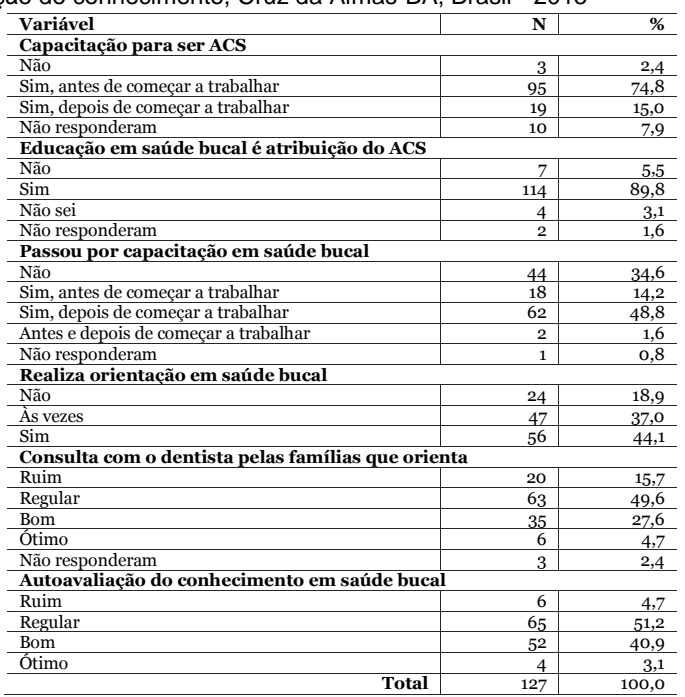

Tabela 4. Conhecimentos dos ACS em saúde bucal, , Cruz da Almas-BA, Brasil -2015

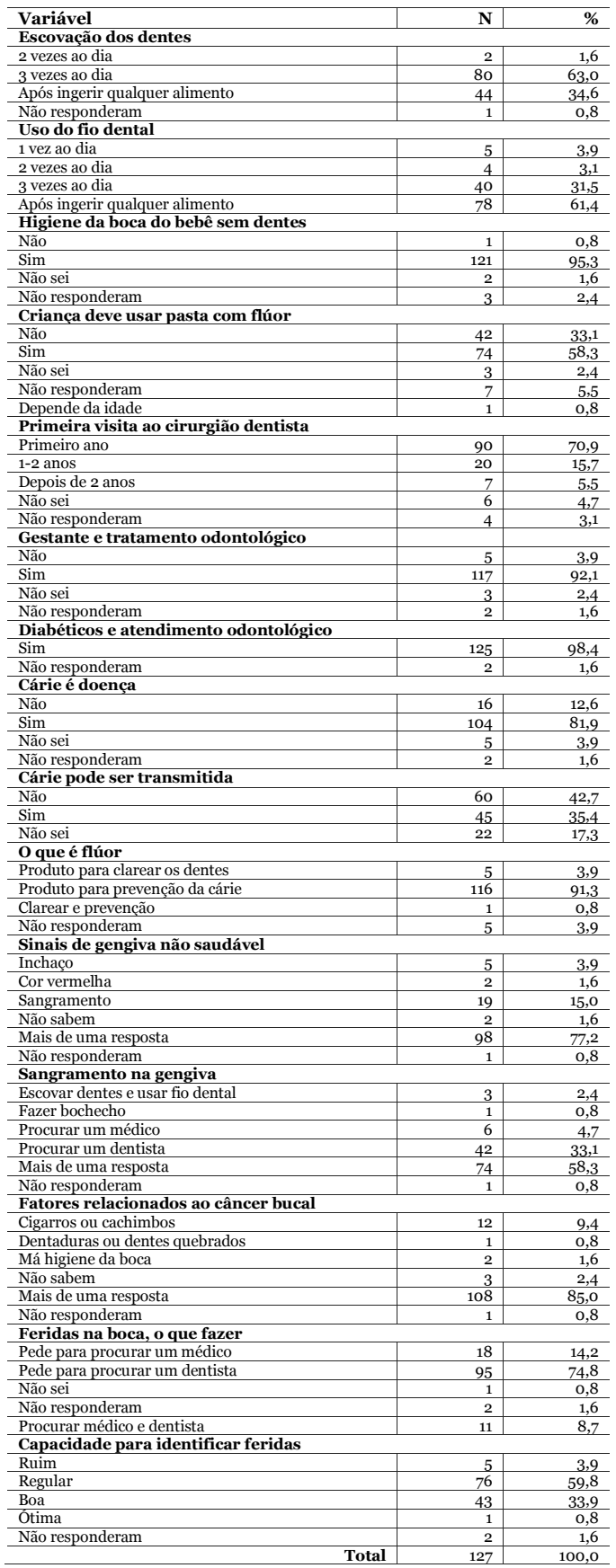


DISCUSSÃO

Nesse estudo, a caracterização socioeconômica dos participantes apontou que a maioria é do sexo feminino e ganha entre um e três salários mínimos, dados semelhantes aos encontrados em estudos realizados em municípios de pequeno porte Piauienses e Mineiros $^{17,18}$. Conforme preconizado pela Lei $\mathrm{n} .{ }^{\circ}$ 10.507 de 10 de julho de $2002^{11}$, que cria a profissão do ACS, a maioria dos agentes desse estudo apresenta o $2^{\circ}$ grau completo, sendo harmonioso com outras localidades ${ }^{17-19}$, o que vai além do exigido. Destaca-se que 18,9\%, desenvolvem ou já concluíram atividades relativas ao ensino superior, apresentando desta forma, interesse em maiores ganhos educacionais e melhorias em seu aprendizado. O fato de apresentarem formação além do preconizado para a profissão torna-se importante, pois demonstra que grande parte dos agentes apresenta certa abertura a apreensão e compreensão de novos conhecimentos $^{17}$ e, portanto, capacitações e melhorias no seu exercício profissional.

O predomínio do acompanhamento de mais de 100 famílias $(78,0 \%)$ e do exercício da profissão há mais de 10 anos (62,2\%) são aspectos positivos quanto à característica de vínculo estabelecido entre estes profissionais e a comunidade assistida pelas USF e UBS. O fato de os ACS serem moradores da comunidade em que atuam ${ }^{11}$ juntamente com o fator tempo e o número de famílias acompanhadas, possibilita-lhes 0 melhor conhecimento dos usuários e a oportunidade de levar às unidades as principais necessidades dos moradores da área adscrita. O curso preparatório para o exercício da profissão também é preconizado pela Lei $n .010 .507$ de 10 de julho de $2002^{11}$, extraindo-se dos resultados que $74,8 \%$ receberam a capacitação adequada antes de iniciar as atividades como ACS.

A compreensão acerca da autopercepção dos agentes em relação a saúde bucal faz-se importante, uma vez que, como concluído em estudo realizado com nove agentes em uma cidade do Paraná ${ }^{20}$, na execução das suas atividades rotineiras, são exigidos por vezes conhecimentos com embasamento científico, bem como saberes e experiências próprias, misturando-se desta forma os saberes popular e médico-científico. Uma vez dominados determinados conhecimentos pelos agentes, estes adentram as casas das famílias durante as visitas, motivando-as a mudanças e melhorias nos seus hábitos diários, refletindo diretamente na saúde e qualidade de vida.
Quanto à autopercepção, os ACS mostraram uma visão crítica quanto sua saúde bucal, uma vez que $86,6 \%$ afirmaram a necessidade de tratamento em seus dentes e gengiva e $64,6 \%$ classificaram a sua saúde bucal como regular. Isso pode ser um indicativo positivo quanto a visão dos agentes em relação a caracterização do que seria uma boa saúde bucal. Esse fator é importante, pois os mesmos realizam o encaminhamento dos usuários às unidades de saúde de acordo com suas necessidades e podem, de forma subjetiva, vir a identificar e encaminhar os pacientes quando for observado que a saúde bucal dos mesmos não se encontra adequada.

Em relação a aparência dos seus dentes e gengiva, os resultados mostraram que para a maioria $(63,8 \%)$, acaba não sendo elemento de interferência nas relações interpessoais cotidianas. O oposto foi encontrado em outros estudos com ACS, que relataram preocupação com a saúde bucal destacando-a e caracterizando-a como imprescindível para concretizar uma identidade, pois o rosto seria o primeiro local para onde se atrai o olhar e por ser o local onde se estampa a identidade de cada $u^{20}$ e que afirmaram ver na saúde bucal um estímulo de convivência social e autoestima $^{18}$.

A saúde bucal é uma área de conhecimento para os agentes, voltada para o seu interesse cotidiano $^{13}$, no que tange, por exemplo, a realização de práticas diárias de higiene bucal, relativas à escovação, interferindo diretamente na sua própria saúde bucal e, portanto, na percepção da necessidade de tratamentos e melhorias e na construção de parte de um conhecimento relativo as suas práticas diárias, que virá a ser repassado durante as visitas.

A maior parte dos agentes considerou que a saúde bucal é uma atribuição do ACS e embora tenham recebido algum tipo de capacitação na área antes ou depois de começar a trabalhar, considerando o seu conhecimento entre regular e bom, grande parte não realiza ou faz de forma esporádica algum tipo de orientação aos usuários. Esses resultados são contrários aos encontrados em outros estudos, em que embora a maior parte dos agentes não tenha recebido nenhum tipo de capacitação ou palestra em saúde bucal ${ }^{8,17}$, realizam atividades ligadas ao tema ${ }^{17}$.

O fato da não realização de atividades básicas em saúde bucal como escovação supervisionada, aplicação de flúor ou instrução para prevenção de doenças como câncer, pode estar ligado a inserção tardia das equipes de 
saúde bucal no $\mathrm{PSF}^{7,17}$, uma vez que, se as atividades e palestras ministradas pelos cirurgiões dentistas aos agentes fosse parte integrante desde a formação do programa, isso seria uma prática comum e os agentes estariam preparados desde o início a levarem a saúde bucal como atividade cotidiana durante as visitas. Outro fator importante pode ser a não realização de atividades de educação continuada na área, de forma que os profissionais da equipe, principalmente os agentes, que estão frequentemente em íntimo contato com as famílias, não se sentem seguros para transmitir informações sobre prevenção de determinadas doenças e orientações básicas de higiene. Para que haja qualidade no trabalho, é essencial que os agentes sejam capacitados, para que assim realizem seu papel de forma segura, transmitindo informações corretas às famílias $^{19}$. A dificuldade em encaminhar os casos com necessidade de tratamento curativo pode ser também uma barreira para a realização de tais atividades ${ }^{8}$.

Segundo o Ministério da Saúde ${ }^{21}$, a higienização da boca e escovação deve ser realizada após cada refeição e também uma última vez antes de dormir, sendo esta complementada com o uso do fio dental, entre todos os dentes. Os agentes não demonstraram conhecimento concordante quanto a realização da escovação e utilização do fio dental, uma vez que poucos afirmaram que a escovação deve ser realizada após ingestão de qualquer alimento, sendo discordante com as respostas dadas sobre a utilização do fio dental.

A saúde bucal de bebês, crianças, gestantes e portadores de doenças sistêmicas como diabetes, deve ser tratada com devida atenção, sendo importante que o agente tenha algum conhecimento na orientação desses grupos às práticas de higiene bucal e encaminhamento para atendimento odontológico, por serem grupos de risco frequentemente encontrados nas visitas domiciliares. A higiene da boca do bebê deve ser realizada com fralda limpa antes mesmo do nascimento dos dentes, assim como as crianças podem fazer uso de dentifrícios fluoretados em pequenas quantidades (menos do que um grão de arroz) desde o irrompimento do primeiro dente $^{21}$. Quanto a essas informações, os agentes demonstraram bom conhecimento, com respostas compatíveis ao recomendado pelo Ministério da Saúde, fato que pode ser explicado pela grande quantidade de campanhas e informações, disponíveis na unidade, presentes em cartazes ou nas próprias "salas de espera" realizadas pelos profissionais da equipe, voltadas a saúde do bebê, da gestante e das crianças, grupos de grande enfoque das equipes.

Existem ainda muitos mitos e impasses ao atendimento odontológico para gestantes e pacientes com alguma alteração sistêmica. As más condições de higiene bucal podem causar doenças bucais que podem agravar determinadas enfermidades como diabetes e doenças cardiovasculares ${ }^{21}$. É comum o tabu de que a gravidez ocasiona cárie e vem a enfraquecer os dentes, como demonstrado em estudo realizado com mulheres grávidas ${ }^{22}$, quando na realidade, isso é atribuído a alta ingestão de alimentos no período, sem a escovação adequada. A presença de doenças bucais durante a gravidez pode levar ao baixo peso do recém-nascido ${ }^{21}$. O dentista, assim como toda equipe, deve ter conhecimento acerca de como deve ser feito o atendimento a esses grupos, sendo importante uma anamnese criteriosa como forma de prevenir possíveis danos. Os ACS desse estudo apresentaram respostas positivas e mostraram-se conscientes quanto ao encaminhamento desses pacientes ao atendimento odontológico, o que também pode ser explicado pelo grande direcionamento de campanhas a esse público nas USF, sendo abordados diversos aspectos para a saúde geral dos mesmos.

Por ser a cárie uma doença frequente e conhecida popularmente, torna-se importante avaliar o conhecimento dos agentes quanto ao desenvolvimento e transmissibilidade da mesma. É importante o conhecimento adequado sobre os múltiplos fatores que levam ao desenvolvimento da cárie, principalmente durante visitas às mães como prevenção para crianças e bebês. Os agentes consideram a cárie como uma doença $(81,9 \%)$, e poucos afirmaram que tal doença pode ser transmitida, frente a um grande número que afirmou não saber ou não acreditar na transmissibilidade. A cárie é uma doença multifatorial, envolvendo aspectos que vão desde uma alimentação rica em açúcares e higiene bucal inadequada, presença de biofilme e com influência do uso do flúor. Juntamente a esses fatores, o desenvolvimento da cárie deve ser atribuído também ao desequilíbrio de bactérias naturalmente presentes na boca.

Vale ressaltar que a cárie é uma doença que tem início com a formação do biofilme dental, é mediada pela dieta, tendo a sacarose (presente em muitos alimentos) amplo papel nas propriedades cariogênicas do biofilme dentário, sendo um fator necessário para seu desenvolvimento ${ }^{22-24}$. O "transmissível" na 
doença cárie é o que se incorpora na rotina da criança, que são os hábitos de higiene e dieta da família e não especificamente os microrganismos que inevitavelmente, farão parte da microbiota oral ${ }^{25}$.

Quando questionados sobre a função do flúor, o resultado obtido foi bastante positivo. A maioria dos ACS respondeu que o flúor é um produto para a prevenção da cárie. Assim, verificou-se que os agentes compreendem a importância do flúor, o que pode somar-se a outro estudo realizado com ACS e mulheres grávidas, em que os resultados apontaram que a população estudada compreende a importância do flúor em todas as fases da vida $^{26}$.

Durante as visitas, a integração dos agentes com as famílias e o vínculo estabelecido é muito importante para auxiliar na promoção da saúde de uma forma geral. A maioria dos agentes relatou sua capacidade para identificar feridas como regular e boa. Este torna-se um fator importante em relação a atenção dos agentes ao discurso dos usuários sobre a sua saúde, bem como a própria observação do estado geral dos indivíduos para possíveis encaminhamentos. O câncer bucal, por exemplo, que normalmente não é identificado pelo próprio paciente, pode ser atribuído a diversas causas. Segundo o Instituto Nacional do Câncer (INCA) ${ }^{27}$ e o Ministério da Saúde ${ }^{21}$, os fatores que podem levar ao câncer de boca são idade superior a 40 anos, vício em cachimbos e cigarros, consumo de álcool, má higiene bucal, próteses dentárias mal adaptadas, dieta rica em gorduras e exposição frequente ao sol sem uso de protetor para os lábios. No presente estudo, a grande maioria dos agentes atribuíram a mais de um fator, sendo eles cigarros ou cachimbos, dentaduras ou dentes quebrados e má higiene da boca, o que reflete o entendimento do câncer como uma doença multifatorial. Em outros estudos, as causas mais apontadas pelos agentes como causadoras do câncer foram excesso de álcool e fumo ${ }^{19}$, próteses mal adaptadas, câmaras de sucção e raízes residuais ${ }^{17}$.

O mínimo conhecimento do ACS em saúde bucal é essencial para a promoção da saúde das famílias que acompanham, principalmente para o correto encaminhamento dos mesmos à USF. Sobre a sua conduta diante de serem informados ou identificarem sangramento na gengiva, poucos afirmaram exclusivamente que pedem para procurar um dentista, a maioria marcou mais de uma alternativa, incluindo fazer bochechos, procurar um médico, escovar os dentes e usar fio dental.
Este dado apresenta-se contrário ao encontrado quando os agentes foram questionados sobre a sua conduta quando observam aumento de volume ou feridas na boca, em que a maioria dos agentes afirmou que pede exclusivamente para procurar atendimento odontológico.

Estes resultados podem ser atribuídos ao fato do sangramento gengival, associado ao acúmulo de placa (gengivite), ser comum entre os pacientes quando estes não realizam a higienização adequada da boca, levando a irrelevância de tal ocorrência por parte dos agentes, que devem aconselhar principalmente a continuação e melhoria da escovação e do uso do fio dental ${ }^{21}$. Isto não ocorre quando uma ferida na boca está presente, pois muitas vezes o paciente reclama de desconforto severo, tanto pela aparência e presença da lesão quanto pela sintomatologia dolorosa que esta pode ocasionar, o que leva o ACS a fazer o encaminhamento correto e imediato do usuário à consulta com o dentista.

CONCLUSÃO

Nesse estudo, observou-se que os ACS apresentaram uma visão crítica do que seria uma boa saúde bucal, percebendo suas necessidades de realização de tratamento odontológico. Tal situação é positiva, visto que esses profissionais podem ter o mesmo olhar durante as visitas, observando a necessidade de tratamento dos usuários e realizando o encaminhamento às unidades.

As capacitações em saúde bucal com maior frequência fazem-se necessárias, pois embora os participantes do estudo tenham mostrado conhecimento considerável quanto a importância do atendimento odontológico a gestantes, crianças e diabéticos e utilização do flúor, o contrário ocorreu para aspectos básicos como escovação e conduta de encaminhamento ao cirurgião dentista.

Um agente mais capacitado compreende as principais necessidades das famílias acompanhadas e se fortalece como um importante instrumento na identificação e prevenção de doenças, além de proporcionar um "feedback" às equipes de saúde bucal quanto ao planejamento dos atendimentos na unidade e domiciliares, temas a serem discutidos nas salas de espera e locais que possam receber palestras e campanhas em saúde bucal, como creches e escolas.

AGRADECIMENTOS

À Fundação de Amparo à Pesquisa do Estado da Bahia (FAPESB) pela concessão da bolsa de Iniciação Científica à primeira autora (№ 4840/2015). 


\section{REFERÊNCIAS}

1. Ministério da Saúde (BR). Política Nacional de Atenção Básica. Brasília: Ministério da Saúde; 2012.

2. Lavras C. Atenção Primária à Saúde e a Organização de Redes Regionais de Atenção à Saúde no Brasil. Saúde Soc. 2011;20(4): 867-74.

3. Melo MB, Brant LC, Oliveira LA, Santos APS. Qualificação de agentes comunitários de saúde: instrumento de inclusão social. Trab Educ Saúde. 2009/2010;7(3):463-77.

4. Ministério da Saúde (BR). Saúde da família: uma estratégia para a reorientação do modelo assistencial. Brasília: Ministério da Saúde; 1997.

5. Brasil. Ministério da Saúde. Lei no 1.886, de 18 de dezembro de 1997. Aprova as normas e diretrizes do programa de agentes comunitários de saúde e do programa de saúde da família. Brasília, DF; 1997. [acesso em 2015 mar. 25]. Disponível em: http://189.28.128.100/dab/docs/ legislacao/portaria1886_18_12_97.pdf.

6. Ministério da Saúde (BR); Fundação Oswaldo Cruz. Saúde da Família. Avaliação da Implementação em Dez Grandes Centros Urbanos. Síntese dos Principais Resultados. $2^{\underline{a}}$ Ed. Brasília, DF; 2005.

7. Brasil. Ministério da Saúde. Portaria MS/GM n.1.444 de 28 de dezembro de 2000. Estabelece incentivo financeiro para a reorganização da atenção básica à saúde da família prestada nos municípios por meio do Programa de Saúde da Família. Diário Oficial da União. 2000 dez. 29; Seção 1, p. 85.

8. Mialhe $F L$, Lefèvre $F$, Lefèvre AMC. O agente comunitário de saúde e suas práticas educativas em saúde bucal: uma avaliação qualiquantitativa. Ciênc saude coletiva. 2011;16(11):4425-32.

9. Brasil. Ministério da Saúde. Portaria no 267, de 06 de março de 2001. Regulamentação da Portaria n. $.1 .444 / G M$, de 28 de dezembro de 2000, que criou o incentivo de saúde bucal destinado ao financiamento de ações e da inserção de profissionais desta área no Programa de Saúde da Família (PSF). Diário Oficial da União. 2001 mar. 07, seção 1, pg. 67.

10. Brasil. Decreto n. -3189 , de 4 de outubro de 1999. Fixa diretrizes para o exercício da atividade de Agente Comunitário de Saúde (ACS) e dá outras providências. Presidência da República, Subchefia para Assuntos Jurídicos. 1999 out. 4 [Acesso em 2015 mar 25]. Disponível em: http://www.planalto.gov.br/ ccivil_03/decreto/D3189.htm.

11. Brasil. Diário Oficial da República Federativa do Brasil, Poder Executivo. Lei n.. 10.507 de 10 de julho de 2002. Cria a Profissão de Agente Comunitário de Saúde e dá outras providências. Brasília, DF; 2002. [acesso em 2015 mar. 25]. Disponível em: http://www.planalto.gov.br/ccivil_03/leis/2002/L 10507.htm.

12. Rosa AS, Caviccioli MGS, Brêtas ACP. O significado que o agente comunitário de saúde atribui ao seu trabalho no processo de construção do Sistema Único de Saúde no Brasil. Acta Paul Enf. 2004;17(3):255-61.

13. Pires ROM, Neto FL, Lopes JB, Bueno SMV. O conhecimento dos agentes comunitários sobre saúde bucal: uma perspectiva sobre deficiências em educação em saúde no PSF. Ciênc saude coletiva. 2007;6(3):325-34.

14. Frazão $P$, Marques $D$. Efetividade de programa de agentes comunitários na promoção da saúde bucal. Rev Saúde Pública. 2009;43(3): 463-71.

15. Instituto Brasileiro de Geografia e EstatísticaIBGE, 2014. [homepage na internet]. [acesso em 2015 mar. 25]. Disponível em: http://cod.ibge.gov.br/234F0.

16. Batista MJ, Rins LB, Gonçalo CS, Miyauchi kubo FM, Amaral RG, Sousa MLR. Treatment needs and self-perception of oral health among adolescents. Rev Gauch Odontol. 2012; 60(3):289-96.

17. Moura MS,Carvalho CJ, Amorim JTC, Marques MFSS, Moura LFAD, Mendes RF. Perfil e práticas de saúde bucal do agente comunitário de saúde em municípios piauienses de pequeno porte. Ciênc saude coletiva. 2010; 15(Supl 1):1487-95.

18. Vasconcelos M, Cardoso AVL, Abreu MHNG. Os desafios dos Agentes Comunitários de Saúde em relação à saúde bucal em um município de pequeno porte. Arq Odontol. 2010;45(2):98-104.

19. Gouvea GR, Silva MAV, Pereira AC, Mialhe FL, Cortellazzi KL, Guerra LM. Avaliação do conhecimento em saúde bucal de agentes comunitários de saúde vinculados a Estratégia Saúde da Família. Ciênc saude coletiva. 2015; 20(4):1185-97.

20. Koyashiki GAK, Souza RAA, Garanhani ML. O trabalho em saúde bucal do Agente Comunitário de Saúde em Unidades de Saúde da Família. Ciênc saude coletiva. 2008;13(4): 1343-54.

21. Ministério da Saúde. Mantenha seu sorriso fazendo a higiene bucal corretamente. Brasília, DF: Ministério da Saúde; 2012.

22. Paes Leme AF, Koo $H$, Bellato $C M$, Bedi $G$, Cury JÁ. The role of sucrose in cariogenic dental biofilm formation-new insight. J Dent Res. 2006;85(10):878-87.

23. Sheiham A, James WPT. Diet and dental caries: the pivotal role of free sugars reemphasized. J Dent Res.2015;94(10):1341-7.

24. Kidd $E$. The implications of the new paradigm of 
dental caries. J Dent. 2011;39(Suppl 2):S3-8.

25. Maia LC, Primo LG. Odontologia integrada na infância. São Paulo: Santos; 2012.

26. Frazão $P$, Marques DSC. Influência de agentes comunitários de saúde na percepção de mulheres e mães sobre conhecimentos de saúde bucal. Ciênc saude coletiva. 2006;11(1): $131-44$

27. Instituto Nacional do Câncer (INCA). Câncer de boca: Fatores de risco [acesso em 20 jul 2016]. Disponível em: http://www.inca.gov.br/ conteudo_view.asp?id=324.

\section{CONFLITO DE INTERESSES}

Os autores declaram não haver conflitos de interesse

\section{AUTOR PARA CORRESPONDÊNCIA}

\section{Cristiane Alves Paz de Carvalho}

Universidade Estadual do Sudoeste da Bahia - UESB, Departamento de Saúde I

Avenida José Moreira Sobrinho, $\mathrm{s} / \mathrm{n}$, Jequiezinho

45208-409 Jequié - BA, Brasil

E-mail: capcarvalho@uesb.edu.br 